\title{
The Whirlpool Sign in Abdominal Pain: The Hernias that Radiologists Should Never Forget
}

\author{
William Andres PM1,2,3,4* and Andrea Osorio N2,4 \\ 1Professor, Fundación Universitaria del Área Adina (FUAN), Colombia \\ ${ }^{2}$ Department of Radiology, Sabana University, Colombia \\ 3Physician, Rosario University, Colombia \\ ${ }^{4}$ Department of Radiology, Hospital universitario de la Samaritana, Colombia
}

\section{Case Report}

Volume 3 Issue 1

Received Date: December 31, 2018

Published Date: January 30, 2019

*Corresponding author: William Andres Prada Mancilla, Professor, Fundación Universitaria del Área Adina (FUAN), Rosario University, Sabana University, Bogotá, Colombia, Tel: 3008719482;

Email: wpradamancilla@gmail.com

\section{Abstract}

An internal hernia is defined as a protrusion of a viscus through a peritoneal or mesenteric opening within the peritoneal cavity, which occurs in less than $1 \%$ of patients with abdominal pain. The diagnostic imaging should be performed with contrast tomography looking for findings of grouping of handles; grouping, elongation and regurgitation of the mesenteric vessels; displacement of the mesenteric main trunk to the right, among others. The role of the radiologist is crucial in the approach of these patients, where a timely decision allows improving the decision making and the prognosis of these patients.

Keywords: Whirlpool Sign; Hernias; Intestinal Obstruction; Abdominal Tomography

\section{Introduction}

Intestinal obstruction can become a diagnostic challenge for the general surgeon. The role of the radiologist in this diagnostic approach, especially when it is not a clear diagnosis, becomes a fundamental role that allows a better approach and long-term results for this type of patients [1]. We present a clinical case of a patient with intestinal obstruction due to an internal hernia and the discussion with the recommendations in the diagnostic approach.

\section{Clinical Case}

This is a 63-year-old patient with a history of appendectomy 8 years ago, who consulted due to intermittent generalized abdominal pain with bilious vomiting, and liquid stools in multiple episodes, since 1 day the patient reports absence of depositions. Physical examination revealed stable vital signs, mild tachycardia and diffuses abdominal pain without clear signs of peritoneal irritation. Ultrasound of the abdomen was requested, presenting a normal result, which led to a contrast-enhanced tomography of the abdomen. Abdominal tomography shows no transition zones or thickening of the intestinal walls, which show clear intestinal obstruction.

As the only important finding is a tortuosity of the mesenteric arterial vessels (whirlpool sign) in relation to an internal hernia associated with the patient's surgical history (Figures $1(\mathrm{a}-\mathrm{d})$ ). The patient is taken to surgery, 


\section{Clinical Radiology \& Imaging Journal}

where they find signs of suffering from thin distal loops around the internal hernia. After 3 days in critical care and two weeks in hospitalization was discharged with satisfactory evolution.

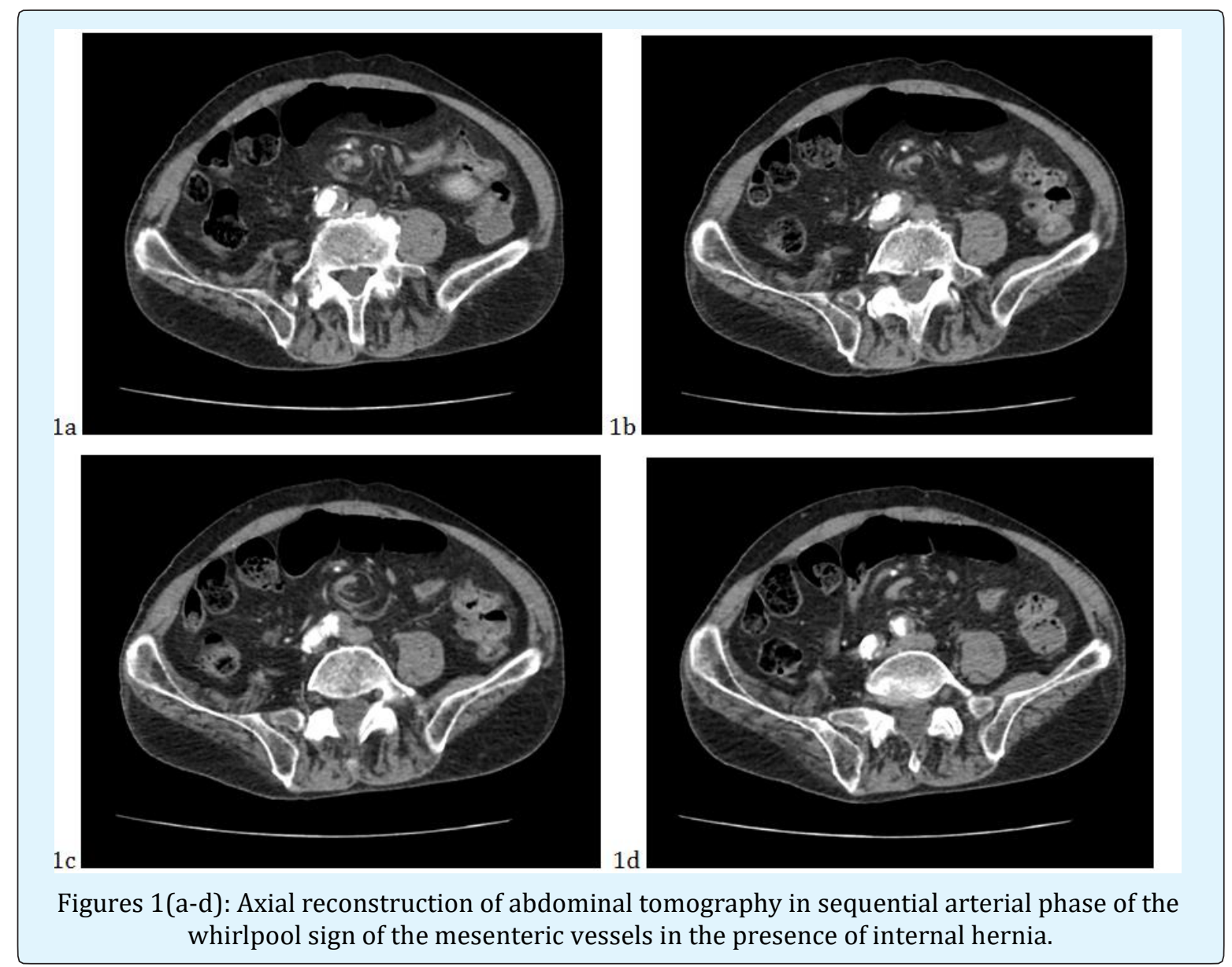

\section{Discussion}

An internal hernia is defined as a protrusion of a viscus through a peritoneal or mesenteric opening within the peritoneal cavity, which leads to its encapsulation within a compartment inside the abdominal cavity. The hernial orifice can be an already existing anatomical structure (congenital, such as foramina, recesses and pits), in which case they do not present a sac, or originate from acquired form after surgery, trauma, inflammation or circulatory problems, in which case there would be a sac herniary [1].

\section{Incidence}

These are quite rare defects, with an overall incidence lower than $1 \%$ of all hernias $(0.5-4 \%)$, between $0.2-5.8 \%$ of all intestinal obstructions, and with mortality that it can reach $50 \%$ if there is intestinal vascular compromise. It is accepted that between $10-50 \%$ could be discovered during an unrelated laparotomy or autopsy [1].

The average frequency of each type of internal hernia is as follows: paraduodenal (53\%), pericecal (13\%), foramen of Winslow (8\%), transmesenteric and transmesocolic (8\%), sigmoid (6\%), transepiploic (1 -4\%) and retroanastomotic (5\%) [1].

\section{Etiopathogenesis}

A part of the intestinal loop enters the hole or defect, stimulates a violent peristalsis of its proximal part, creates an effect of gas entrapment and due to the effect of the severity of the weight itself a distension develops, all of which increases the segment of handle extruded through the ring until the handle rotates around a redundant meso and turns [1]. 


\section{Clinical Radiology \& Imaging Journal}

\section{Diagnosis}

Clinical: Since the herniation is usually intermittent, in many cases patients remain asymptomatic for a large part of their lives. In other cases, they present a history of episodes of recurrent postprandial pain, such as discomfort accompanied by a sensation of distension, nausea and vomiting, especially after a copious meal. In the extreme case, the clinical progress until reaching the symptoms and signs typical of a complete intestinal obstruction. An internal hernia should be suspected in all patients with signs of intestinal obstruction, in the absence of inflammatory bowel disease, external hernia or previous surgery [1].

Diagnostic Images: The pre-surgical diagnosis of internal hernia is difficult. The following findings in the tomography are associated, are relevant and are considered as anatomical markers in the identification of the internal hernia: 1) Grouping of handles; 2) Grouping, elongation and regurgitation of the mesenteric vessels; 3 ) Displacement of the mesenteric main trunk to the right; 4) Abnormal localization of an intestinal segment in a susceptible region such as the minor sac; 5) Encapsulation and fixed accumulation of intestinal loops in the peritoneal cavity; 6) Dilated intestinal loop swirl, altered and displaced vascular pedicle and converging vessels of the hernia defect; 7) Stasis or lack of progression of the intraluminal contrast medium; 8) Signs of intestinal obstruction $[2,3]$.

The role of ultrasound in the evaluation of the whirlwind sign is limited to the evaluation of the orientation of the mesenteric vessels (superior mesenteric artery and vein). It is a dynamic study, without radiation, where we can evaluate in real time the behavior of the mesenteric vessels and the physical characteristics of their intravascular flow should be evaluated in the window for evaluation of the head of the pancreas where the artery is more medial and the vein more lateral. Likewise, the basic flow of the vein and the three-phase flow of the superior mesenteric artery should be evaluated when this is reversed it is known as the whirlpool sign. Depending on the age group, the most likely etiologic diagnosis will be different. Ultrasound is more useful in children and newborns. When the positive predictive value for intestinal malrotation is present, it is very high [4]. In adults, depending on the surgical history, the whirlpool sign is an indirect sign and usually requires additional studies, such as tomography [4].

\section{Conclusion and Recommendations in the Diagnostic Approach}

The whirlpool sign in the contrasted tomography of the abdomen is presented very infrequently; however, it should always be looked for in the approach of the diagnosis of the patient with abdominal pain. The recommendations for the approach in tomography should always be in the arterial phase of the study where the anatomy of the mesenteric vessels is best evaluated throughout their course and inflow. In case of having an ultrasound assessment opportunity, the approach should always be earlier in the window for assessment of the pancreas, evaluating in the Doppler color study and power the characteristic venous and arterial patterns. When we are confronted as radiologists with this type of pathology, we must report the anatomical location, the vascular compromise that may occur, if there is a compromise of associated bowel loops, and the physiopathological mechanism of torsion of the vessels with the use of the contrast medium at the right time (arterial) [5].

\section{References}

1. Fernando CT, Alfredo ME (2012) Hernias abdominales internas. Eventraciones. Otras hernias de pared y cavidad abdominal 57: 693-694.

2. Motta-Ramírez GA, Alonso-Blancas E, González Merino LI, Montero MJC (2012) La hernia interna como condicionante del síndrome de obstrucción intestinal: identificación y evaluación por tomografía computada multidetector. Anales de Radiología México 3: 157-164.

3. Dinesh S, Ashish C (2016) The "abdominal whirlpool" sign. Abdominal radiology 41(7): 1437-1438.

4. Zhang W, Sun H, Luo F (2017) The efficiency of sonography in diagnosing volvulus in neonates with suspected intestinal malrotation. Medicine (Baltimore) 96(42): e8287.

5. Artul S, Nijim Y, Abu Rahmah Y, Habib G (2014) Whirlpool sign. BMJ case reports.

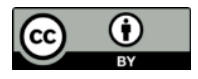

